\title{
Residuos hospitalarios peligrosos en un centro de alta complejidad
}

\author{
Alejandra Neveu C ${ }^{1 a}$, Patricia Matus $C^{2}$. \\ Management of hazardous waste \\ in a hospital
}

Background: An inadequate management of hospital waste, that have toxic, infectious and chemical wastes, is a risk factor for humans and environment. Aim: To identify, quantify and assess the risk associated to the management of hospital residues. Material and methods: A cross sectional assessment of the generation of hazardous waste from a hospital, between June and August 2005, was performed. The environmental risk associated to the management of non-radioactive hospital waste was assessed and the main problems related to solid waste were identified. Results: The rate of generation of hazardous non-radioactive waste was 1.35 tons per months or $0.7 \mathrm{~kg} / \mathrm{bed} / \mathrm{day}$. Twenty five percent of hazardous liquid waste were drained directly to the sewage system. The drug preparation unit of the pharmacy had the higher environmental risk associated to the generation of hazardous waste. The internal transport of hazardous waste had a high risk due to the lack of trip planning. The lack of training of personnel dealing with these waste was another risk factor. Conclusions: Considering that an adequate management of hospital waste should minimize risks for patients, the hospital that was evaluated lacks an integral management system for its waste (Rev Méd Chile 2007; 135: 885-95).

(Key words: Hazardous waste; Hospital materials management; Medical waste)

Recibido el 31 de agosto, 2006. Aceptado el 8 de enero, 2007.

${ }^{1}$ CIMMT \& S S.A. (Centro de Investigación de Minería y Metalurgia). ${ }^{2}$ Unidad de Salud y Ambiente, Centro Nacional del Medio Ambiente, Universidad de Chile. Santiago, Chile.

angeniero Industrial, Magíster en Gestión y Planificación Ambiental.

L os problemas asociados a los residuos generados por los centros hospitalarios, han sido motivo de preocupación internacional. Dicha motivación ocurre debido al amplio espectro de peligrosidad, comprendiendo desde la potencial propagación de enfermedades infecciosas, hasta riesgos ambientales derivados de los métodos empleados para su tratamiento y disposición final. Es por ello que la problemática ha trascendido el

Correspondencia a: Dra. Patricia Matus C. Centro Nacional del Medio Ambiente de la Universidad de Chile. Avenida Larraín 9975. Fax: (562) 2751688. E mail: pmatus@cenma.cl campo técnico sanitario y ha involucrado aspectos sociales, económicos, políticos y ambientales ${ }^{1}$. El mal manejo de los residuos hospitalarios representa un riesgo para las personas y el medio ambiente por la presencia de residuos infecciosos, tóxicos, químicos y objetos cortopunzantes $\mathrm{y}$, principalmente, provoca gran inquietud y percepción de riesgo en la población general ${ }^{2,3}$.

La Organización Mundial de la Salud identificó los potenciales riesgos del manejo de residuos hospitalarios peligrosos, listando los siguientes efectos potenciales: SIDA, hepatitis B y C, infecciones gastroentéricas; infecciones respiratorias; infecciones dérmicas e intoxicaciones, entre otras 
patologías ${ }^{4}$. Estudios realizados en la década 1990-99 en Chile, señalaron que los residuos hospitalarios correspondían a 29.330 toneladas/ año de las cuales $80 \%$ se concentraba en la Región Metropolitana (CONAMA, 1994). En la actualidad no se cuenta con una norma específica que regule el adecuado manejo de este tipo de residuos, si bien el Ministerio de Salud se encuentra estudiando un reglamento sobre la materia ${ }^{5}$, sólo existen instructivos de carácter indicativo. El presente estudio tiene por finalidad aportar al conocimiento del tipo y cantidad de residuos generados en un centro de alta complejidad, realizar un análisis del manejo de los residuos durante su ciclo comprendido desde su generación hasta su entrega a un destinatario o empresa que lo dispone posteriormente. Con miras a evaluar el riesgo ambiental presente, identificando aquellas áreas y procedimientos más riesgosos, a modo de aportar al mejoramiento del sistema de manejo actualmente imperante en un instituto de referencia nacional.

\section{MATERIAL Y MÉTODO}

Se realizó un estudio de tipo corte transversal entre junio y agosto de 2005 en el Instituto, en Santiago de Chile, dentro del marco de una tesis para optar al grado de Magíster en Gestión Ambiental del autor principal de este estudio.

Dicho estudio contempló la observación en terreno del manejo de los residuos hospitalarios, con la medición directa por medio de los volúmenes alcanzados en las bolsas y recipientes de almacenamiento transitorio de los mismos, en las distintas unidades clínicas y de apoyo evaluadas. Se confeccionó un índice de generación por cantidad de camas, por día y por camas ocupadas. Al no contarse con una clasificación universal, ni un reglamento nacional sobre residuos hospitalarios, se trabajó bajo el criterio denominado de gestión avanzada, que cataloga a los residuos en dos tipos. Residuos tipo I, son aquellos que no pueden eliminarse mediante sistemas de descarte de residuos urbanos, como lo son los residuos infecciosos, patológicos, cortopunzantes, farmacéuticos, genotóxicos, químicos peligrosos, contenedores presurizados, radioactivos, ecotóxicos. Residuos tipo II, son aquellos que pueden ser eliminados mediante los sistemas de residuos sólidos urbanos, que pueden ser biodegradables, reciclables e inertes (Mery $F$, Tesis doctoral. Universidad Politécnica, Madrid, 1998). El presente reporte se centrará en los hallazgos correspondientes a los residuos catalogados tipo I. Los residuos hospitalarios y hospitalarios peligrosos son los definidos por las guías de la Organización Mundial de la Salud 4 .

Un profesional del área de la ingeniería aplicó una encuesta de evaluación a un total de 17 unidades del Instituto descritas en la Tabla 1. Los resultados fueron registrados y tabulados por el mismo profesional.

La escala de riesgo utilizada en el estudio se basó en el proyecto de Norma Experimental sobre Análisis y Evaluación del Riesgo Medioambiental (AENOR, 2002) y la Directiva Seveso II de la Unión Europea ${ }^{6}$. Se ajustaron los criterios a la realidad nacional mediante opinión de expertos del Instituto, los autores y la directora de la tesis.

La definición de riesgo ambiental usada es toda circunstancia o factor que conlleva la probabilidad de un daño para el medio ambiente» $\gg$.

\section{RESUlTADOs}

El Instituto gestiona, en la actualidad, sus residuos a través de tres modalidades: utiliza personal interno al que le asigna tareas de recolección de residuos tipo II y de material para reciclaje (papel); una empresa externa que se encarga del mantenimiento general de la institución, como es la limpieza de áreas públicas y oficinas; y para el manejo de los residuos tipo I, contrata los servicios de otra empresa externa. Dicha empresa, al menos una vez por semana, dispone de personal que, acompañado por personal interno del Instituto, realiza la recolección de los residuos tipo I ubicados en almacenes temporales destinados para tal fin. Ella suministra los contenedores y bolsas rojas para la recolección y almacenamiento de los residuos peligrosos.

El Instituto no dispone de un Manual de Procedimientos para el Manejo de Residuos Sólidos en sus dependencias, ni de un registro de los residuos generados, por lo que los responsables del manejo de los residuos desconocen las cantidades exactas de residuos producidas. Los residuos cortopunzantes generados, a pesar de estar clasificados por organis- 
Tabla 1. Unidades o centros de responsabilidad estudiados, 2005

\begin{tabular}{|rlcr|}
\hline & Unidades/subunidades & Tipo de unidades & Personal asignado \\
\hline 1 & Acupuntura & Apoyo clínico & 1 \\
2 & Alimentación & Apoyo logístico & 20 \\
3 & Anatomía patológica & Apoyo clínico & 9 \\
4 & Braquiterapia & Clínico & 7 \\
5 & Cirugía (quirófano) & Clínico & 7 \\
6 & Cirugía menor & Clínico & 8 \\
7 & Endoscopia & Clínico & 8 \\
8 & Esterilización & Apoyo clínico & 7 \\
9 & Farmacia* & & \\
& Farmacia central & Apoyo clínico & 11 \\
& Farmacia unidad de dispensación & Apoyo clínico & 2 \\
& Farmacia preparación drogas oncológicas & Apoyo clínico & 2 \\
10 & Farmacia preparación drogas & Apoyo clínico & 1 \\
11 & Imagenologín mujeres & Clínico & 4 \\
12 & Intermedio & Apoyo clínico & 10 \\
13 & Laboratorio & Clínico & 16 \\
14 & Policlínico & Apoyo clínico & 8 \\
15 & Quimioterapia & Clínico & 18 \\
16 & Quimioterapia ambulatoria & Clínico & 8 \\
17 & Radioterapia & Clínico & 3 \\
\end{tabular}

* Farmacia se divide en 4 subunidades las cuales se encuentran físicamente separadas, por lo que se estudiaron de manera independiente.

mos internacionales como peligrosos (Mery F, Tesis doctoral. Universidad Politécnica, Madrid, 1998), son descartados por el Instituto mezclados con los residuos asimilables a los residuos domésticos (tipo II). Sin embargo, es necesario aclarar que ellos se eliminan en cajas (rígidas), que cumplen con estándares de seguridad para los posibles manipuladores. Según el tipo de residuo, el descarte puede realizarse a través de empresas privadas, alcantarillado, atmósfera o por recolección municipal (Tabla 2). Los tipos de residuos por unidades generadoras se describen en la Tabla 3. La cantidad de residuos tipo I se aprecia en la Tabla 4. La segregación en origen se realiza en todas las unidades estudiadas, usándose distintos tipos de contenedores según las caractenísticas físicas del residuo.

El Instituto cuenta con áreas destinadas a almacenar temporalmente tanto residuos tipo I como tipo II, mientras esperan su recolección. La Tabla 5 describe las características de dichas áreas, destaca que sólo el área de pensionado tiene un área exclusiva para el almacenamiento temporal de residuos, claramente señalada.

El transporte interno de los residuos es uno de los aspectos críticos a evaluar y se consideraron las condiciones del recolector (Tabla 6) y las características propias del recorrido (Tabla 7).

El Instituto no dispone de un lugar centralizado para los residuos tipo I, pues se retiran con una frecuencia tal que evita que se acumulen en el Instituto, ésta puede llegar a ser varias veces a la semana.

Se determinó el riesgo ambiental para las unidades generadoras de residuos tipo I (Tabla 8) y para los procedimientos de transporte interno y almacenamiento. La unidad de farmacia, en su subunidad de preparación de drogas, fue la que presentó el riesgo más elevado. Las unidades de anatomía patológica, de hospitalización de mujeres y la unidad de farmacia central presentaron un riesgo 
Tabla 2. M anejo actual de los residuos generados, 2005

\begin{tabular}{|c|c|c|}
\hline Tipo de residuos & Descripción & Manejo actual \\
\hline Residuos patológicos & $\begin{array}{l}\text { Tejidos, órganos, partes del } \\
\text { cuerpo, cadáveres, sangre y otros } \\
\text { fluidos corporales. }\end{array}$ & $\begin{array}{l}\text { Los residuos patológicos a excepción } \\
\text { de sangre y fluidos corporales generados } \\
\text { en anatomía patológica son gestionados } \\
\text { por empresa externa. }\end{array}$ \\
\hline Residuos infecciosos & $\begin{array}{l}\text { Desechos capaces de producir } \\
\text { una enfermedad infecciosa, entre } \\
\text { los que se encuentran los residuos } \\
\text { de aislamiento, y almacenamiento } \\
\text { de agentes infecciosos en alta } \\
\text { concentración, sangre humana y } \\
\text { productos derivados de la sangre, } \\
\text { residuos patológicos, objetos } \\
\text { punzantes contaminados }\end{array}$ & $\begin{array}{l}\text { Los pacientes se manejan como } \\
\text { potencialmente infecciosos, por lo que } \\
\text { se toman medidas precautorias en } \\
\text { cuanto a seguridad personal. } \\
\text { Los residuos generados por los mismos } \\
\text { se manejan como residuos TIPO II, se } \\
\text { descartan por alcantarillado } \\
\text { o en bolsas negras. } \\
\text { Los residuos cortopunzantes van a los } \\
\text { contenedores destinados para tal fin. } \\
\text { Los fluidos corporales se desechan } \\
\text { por alcantarillado. }\end{array}$ \\
\hline $\begin{array}{l}\text { Residuos } \\
\text { cortopunzantes }\end{array}$ & $\begin{array}{l}\text { Agujas, agujas hipodérmicas, hojas de } \\
\text { escalpelos, sets de infusión, cuchillos, } \\
\text { filos, pedazos de vidrio, clavos y cualquier } \\
\text { otro elemento que cuente con esas } \\
\text { propiedades. }\end{array}$ & $\begin{array}{l}\text { Se recolectan en contenedores } \\
\text { de cartón destinados para tal } \\
\text { fin, al llenarse } 3 / 4 \text { partes los } \\
\text { mismos son sellados y desechados } \\
\text { como residuos TIPO II. }\end{array}$ \\
\hline $\begin{array}{l}\text { Residuos } \\
\text { farmacéuticos }\end{array}$ & $\begin{array}{l}\text { Productos farmacéuticos vencidos, } \\
\text { no usados, derramados y contaminados, } \\
\text { drogas y sueros que ya no son necesarios. } \\
\text { Elementos descartados utilizados para la } \\
\text { aplicación de sustancias farmacéuticas } \\
\text { como botellas o frascos con restos, guantes, } \\
\text { máscaras, tubos de conexión. }\end{array}$ & $\begin{array}{l}\text { Fármacos gestionados } \\
\text { por empresa externa. } \\
\text { Botellas de vidrios son reutilizadas. } \\
\text { Guantes, máscaras y otros son } \\
\text { tratados como residuos TIPO II. }\end{array}$ \\
\hline $\begin{array}{l}\text { Contenedores } \\
\text { presurizados }\end{array}$ & $\begin{array}{l}\text { Cilindros de gas comprimido, cartuchos } \\
\text { y latas de aerosol. Gases anestésicos, } \\
\text { óxido de etileno, oxígeno, } \\
\text { aire comprimido. }\end{array}$ & $\begin{array}{l}\text { Los cartuchos de óxido de } \\
\text { etileno, latas de aerosol son } \\
\text { desechados como residuo TIPO II. }\end{array}$ \\
\hline Residuos genotóxicos & $\begin{array}{l}\text { Drogas citotóxicas. } \\
\text { Vómitos, orina o excrementos de } \\
\text { pacientes tratados con drogas citotóxicas. } \\
\text { Material contaminado de la preparación } \\
\text { y administración de drogas como jeringas, } \\
\text { agujas, envoltorios, matraces, bajadas, } \\
\text { elementos de medición. } \\
\text { Drogas vencidas, soluciones sobrantes }\end{array}$ & $\begin{array}{l}\text { El material contaminado de la } \\
\text { preparación y administración } \\
\text { de drogas es gestionado } \\
\text { por empresa externa. } \\
\text { Los fluidos corporales se } \\
\text { desechan por alcantarillado. }\end{array}$ \\
\hline
\end{tabular}




\begin{tabular}{|c|c|c|}
\hline Tipo de residuos & Descripción & Manejo actual \\
\hline Residuos químicos & $\begin{array}{l}\text { Desechos de procedimientos de } \\
\text { limpieza, desinfección y } \\
\text { mantenimiento. } \\
\text { Formaldehído, xileno, químicos } \\
\text { fotográficos, solventes, químicos } \\
\text { orgánicos, químicos inorgánicos. } \\
\text { Residuos de la fabricación de } \\
\text { moldes (cerrobend) para los } \\
\text { campos de irradiación. }\end{array}$ & $\begin{array}{l}\text { Todos se desechan por } \\
\text { alcantarillado, a excepción del } \\
\text { fijador en imagenología, el } \\
\text { cual es retirado por empresa } \\
\text { externa para la posterior } \\
\text { recuperación de plata. } \\
\text { Residuos de cerrobend como } \\
\text { residuos TIPO II y } \\
\text { alcantarillado. }\end{array}$ \\
\hline Residuos radioactivos & $\begin{array}{l}\text { Desechos contaminados con radio } \\
\text { nucleótidos. }\end{array}$ & $\begin{array}{l}\text { Retirados por la Comisión } \\
\text { Chilena de Energía Nuclear. }\end{array}$ \\
\hline Residuos ecotóxicos & $\begin{array}{l}\text { Formaldehído, xileno químicos } \\
\text { fotográficos, solventes. Residuos } \\
\text { con alto contenido de metales pesados. } \\
\text { Drogas citotóxicas, desechos } \\
\text { biológicos de pacientes tratados } \\
\text { con drogas citotóxicas, material } \\
\text { contaminado de la preparación y } \\
\text { administración de drogas. } \\
\text { Oxido de etileno. } \\
\text { Productos farmacéuticos vencidos, } \\
\text { no usados, derramados y contaminados. } \\
\text { Desechos contaminados con radio } \\
\text { nucleótidos. }\end{array}$ & $\begin{array}{l}\text { Fijador en imagenología } \\
\text { retirado por empresa externa } \\
\text { Fijador de radioterapia, } \\
\text { formaldehído, xileno se } \\
\text { desechan por alcantarillado. } \\
\text { Drogas citotóxicas, productos } \\
\text { farmacéuticos, material } \\
\text { contaminado de la preparación } \\
\text { y administración de drogas } \\
\text { retiradas por empresa externa. } \\
\text { Radio nucleótidos retirados por } \\
\text { la Comisión Chilena de Energía Nuclear. } \\
\text { Oxido de etileno a la atmósfera. }\end{array}$ \\
\hline $\begin{array}{l}\text { Residuos generales } \\
\text { (TIPO II) }\end{array}$ & $\begin{array}{l}\text { Son similares a los domiciliarios siempre } \\
\text { y cuando estén separados en el punto } \\
\text { de origen de los residuos clasificados } \\
\text { como potencialmente peligrosos. }\end{array}$ & $\begin{array}{l}\text { Vehículos de recolección } \\
\text { municipal diaria. }\end{array}$ \\
\hline
\end{tabular}

Fuente de la clasificación del tipo de residuo referencia 4 y de la descripción del manejo, referencia 1.

medio. Al ser todas las áreas de almacenamiento improvisadas, sin señalización y de fácil acceso, este procedimiento presentó el mayor riesgo. El transporte interno de residuos tipo I, también presentó un riesgo ambiental acumulado alto, principalmente por la ruta utilizada, la ausencia de planificación de los recomidos, los horarios usados y la falta de capacitación del personal (Tabla 7).

Con respecto al modelo de manejo actual de los residuos, se caracteriza por que el flujo se divide en peligrosos (tipo I) y no peligrosos (tipo II) (Figura 1).

Los residuos no peligrosos son generados en distintas unidades y almacenados temporalmente en ella; posteriormente son recolectados y trasladados hacia un área destinada a almacenamiento final (dentro del perímetro de la institución), donde esperan la recolección por parte de la municipalidad. En el proceso se detectan posibles pérdidas que implica riesgo de contaminación ambiental y riesgo para la salud de las personas.

En cuanto al tipo de residuos peligrosos, actualmente, se segregan residuos farmacéuticos (material contaminado de preparación y administración de drogas, medicamentos caducados), cortopunzantes, patológicos (material orgánico) y químicos (fijador-revelador). El resto de los residuos tipo I sólidos son descartados conjuntamente 
Tabla 3. Generación de residuos por unidades en estudio, 2005

\begin{tabular}{|c|c|c|}
\hline Tipo de residuos & Unidades generadoras & \\
\hline Residuos infecciosos & $\begin{array}{l}\text { Quimioterapia - Hospitalización } \\
\text { Quimioterapia ambulatoria } \\
\text { Cuidados intermedios } \\
\text { Hospitalización mujeres } \\
\text { Anatomía patológica }\end{array}$ & $\begin{array}{l}\text { Policlínicos } \\
\text { Radioterapia } \\
\text { Endoscopia } \\
\text { Cirugía (menor/quirófano) } \\
\text { Laboratorio }\end{array}$ \\
\hline Residuos patológicos & $\begin{array}{l}\text { Laboratorio } \\
\text { Cirugía (menor/quirófano) } \\
\text { Anatomía patológica }\end{array}$ & \\
\hline Residuos genotóxicos & $\begin{array}{l}\text { Quimioterapia - Hospitalización } \\
\text { Quimioterapia ambulatoria } \\
\text { Cuidados intermedios } \\
\text { Hospitalización mujeres } \\
\text { Cirugía (menor/quirófano) } \\
\text { Anatomía patológica } \\
\text { Imagenología }\end{array}$ & $\begin{array}{l}\text { Farmacia } \\
\text { Laboratorio } \\
\text { Braquiterapia } \\
\text { Esterilización } \\
\text { Policlínicos } \\
\text { Radioterapia } \\
\text { Endoscopia }\end{array}$ \\
\hline Residuos ecotóxicos & $\begin{array}{l}\text { Quimioterapia - Hospitalización } \\
\text { Quimioterapia ambulatoria } \\
\text { Cuidados intermedios } \\
\text { Hospitalización mujeres } \\
\text { Cirugía (menor/quirófano) } \\
\text { Anatomía patológica } \\
\text { Imagenología }\end{array}$ & $\begin{array}{l}\text { Farmacia } \\
\text { Laboratorio } \\
\text { Alimentación } \\
\text { Braquiterapia } \\
\text { Esterilización } \\
\text { Policlínicos } \\
\text { Radioterapia } \\
\text { Endoscopia }\end{array}$ \\
\hline Residuos cortopunzantes & $\begin{array}{l}\text { Quimioterapia - Hospitalización } \\
\text { Quimioterapia ambulatoria } \\
\text { Cuidados intermedios } \\
\text { Hospitalización mujeres } \\
\text { Anatomía patológica } \\
\text { Cirugía (menor/ quirófano) }\end{array}$ & $\begin{array}{l}\text { Farmacia } \\
\text { Laboratorio } \\
\text { Braquiterapia } \\
\text { Policlínicos } \\
\text { Radioterapia } \\
\text { Acupuntura } \\
\text { Endoscopia }\end{array}$ \\
\hline Residuos farmacéuticos & $\begin{array}{l}\text { Quimioterapia - Hospitalización } \\
\text { Farmacia }\end{array}$ & \\
\hline Contenedores presurizados & $\begin{array}{l}\text { Cuidados intermedios } \\
\text { Hospitalización mujeres } \\
\text { Cirugía (menor/ quirófano) }\end{array}$ & \\
\hline Residuos químicos & $\begin{array}{l}\text { Quimioterapia - Hospitalización } \\
\text { Quimioterapia ambulatoria } \\
\text { Cuidados intermedios } \\
\text { Hospitalización mujeres } \\
\text { Cirugía (menor/quirófano) } \\
\text { Anatomía patológica } \\
\text { Imagenología }\end{array}$ & $\begin{array}{l}\text { Farmacia } \\
\text { Laboratorio } \\
\text { Alimentación } \\
\text { Braquiterapia } \\
\text { Esterilización } \\
\text { Policlínicos } \\
\text { Radioterapia } \\
\text { Endoscopia }\end{array}$ \\
\hline Residuos radioactivos & Radioterapia & Braquiterapia \\
\hline Residuos generales & $\begin{array}{l}\text { Quimioterapia - Hospitalización } \\
\text { Quimioterapia ambulatoria } \\
\text { Cuidados intermedios } \\
\text { Hospitalización mujeres } \\
\text { Cirugía (menor/ quirófano) } \\
\text { Anatomía patológica } \\
\text { Imagenología } \\
\text { Acupuntura }\end{array}$ & $\begin{array}{l}\text { Farmacia } \\
\text { Laboratorio } \\
\text { Alimentación } \\
\text { Braquiterapia } \\
\text { Esterilización } \\
\text { Policlínicos } \\
\text { Radioterapia } \\
\text { Endoscopia }\end{array}$ \\
\hline
\end{tabular}

Fuente clasificación residuo ref. 4. 
Tabla 4. Estimación diaria de generación de residuos tipo l, 2005

\begin{tabular}{|llr|}
\hline Descarte & Tipo de residuo & $\begin{array}{r}\mathrm{kg} / \mathrm{día} \\
\text { (aprox.) }\end{array}$ \\
\hline Bolsas rojas & Material contaminado por preparación y & 6 \\
& administración de drogas. & \\
& Medicamentos caducados o devueltos. & \\
& Tejidos, órganos, partes del cuerpo. & 7 \\
Cajas cartón & Material cortopunzante & \\
Bolsas negras & Medicamentos caducados & \\
& Vías venosas, equipos flevoclisis, & \\
& equipo microgoteo contaminado con drogas & \\
& Contenedores presurizados & \\
& Virutas cerrobend & \\
Parafina sólida & \\
Alcantarillado & Gasas, compresas, apósitos utilizados en cirugía & \\
& Alcohol etílico & 2 \\
Bidones & Formaldehído & 45 \\
Estimación de generación diaria & 0,45 \\
Estimación de generación diaria por cama & 0,7 \\
Estimación de generación diaria por cama ocupada & \\
\hline
\end{tabular}

Tabla 5. U bicación del almacenamiento temporal actual, residuos tipo I, 2005

\begin{tabular}{|c|c|}
\hline Unidades & Ubicación \\
\hline Quimioterapia-hospitalización & $\begin{array}{l}\text { Ubicada al final de pasillo, no cumple con las } \\
\text { condiciones mínimas de seguridad, zona de libre } \\
\text { tránsito y usos múltiples. Área no identificada. }\end{array}$ \\
\hline $\begin{array}{l}\text { Farmacia central } \\
\text { Farmacia preparación drogas } \\
\text { oncológicas } \\
\text { Quimioterapia ambulatoria }\end{array}$ & $\begin{array}{l}\text { Ubicada en baño de pacientes, sin ventilación, no } \\
\text { cumple con las condiciones mínimas de seguridad, } \\
\text { zona de libre tránsito y usos múltiples. Área no } \\
\text { identificada. }\end{array}$ \\
\hline Imagenología & $\begin{array}{l}\text { Ubicada en área de revelado, acceso sólo a personal de } \\
\text { la unidad, sin ventilación. Área no identificada. }\end{array}$ \\
\hline Anatomía patológica & $\begin{array}{l}\text { Contenedores ubicados a la intemperie } \\
\text { (estacionamiento), no cumple con las condiciones } \\
\text { mínimas de seguridad e higiene. Área no identificada. }\end{array}$ \\
\hline Unidad del dolor & Ubicado en balcón, no exclusiva. Área no identificada \\
\hline Pensionado & Área exclusiva, al interior de mueble. Área identificada \\
\hline
\end{tabular}


Tabla 6. Transporte interno - C ondiciones del recolector, 2005

\begin{tabular}{|lcllc|}
\hline Servicio & $\begin{array}{l}\text { Personal } \\
\text { asignado }\end{array}$ & $\begin{array}{l}\text { Protección } \\
\text { personal }\end{array}$ & Capacitación & $\begin{array}{l}\text { Rotación } \\
\text { personal }\end{array}$ \\
\hline $\begin{array}{l}\text { Servicios generales } \\
\text { (interno) }\end{array}$ & 3 & $\begin{array}{l}\text { Guantes, } \\
\text { delantal }\end{array}$ & $\begin{array}{l}\text { Bioseguridad, infecciones intra- } \\
\text { hospitalarias, prevención de riesgos }\end{array}$ & baja \\
Empresa externa & 8 & $\begin{array}{l}\text { Guantes, } \\
\text { delantal }\end{array}$ & $\begin{array}{l}\text { Inducción sobre labores, experiencias } \\
\text { anteriores }\end{array}$ & baja \\
Empresa externa & 1 & $\begin{array}{l}\text { Guantes, } \\
\text { delantal, } \\
\text { mascarilla }\end{array}$ & No existe & baja \\
Empresa externa & 2 & $\begin{array}{l}\text { Guantes, } \\
\text { delantal, } \\
\text { mascarilla }\end{array}$ & $\begin{array}{l}\text { Charlas sobre comportamiento en los } \\
\text { centros de recolección, residuos } \\
\text { transportados, protección personal }\end{array}$ & baja \\
\hline
\end{tabular}

con los residuos tipo II, por lo que siguen su proceso. Los residuos tipo I líquidos son descartados por el alcantarillado y los residuos tipo I gaseosos son descartados directamente a la atmósfera, lo que genera un riesgo de contaminación al ambiente y a la salud de las personas. Los residuos farmacéuticos y patológicos segregados son controlados al momento de la separación y mediante documentación exigida a la empresa recolectora; en el resto del proceso pueden presentar fugas o pérdidas que conllevarían riesgos de contaminación ambiental y riesgo para la salud de las personas. Los residuos químicos peligrosos sólo son controlados en la segregación, el resto del proceso presentaría fugas y se desconoce si la empresa recolectora cuenta con los permisos para su tratamiento, lo que implicaría riesgos potenciales de contaminación ambiental y a la salud de las personas. Los residuos cortopunzantes son recolectados conjuntamente con los residuos tipo II incorporándose en su proceso.

En general, durante todo el proceso se pueden generar pérdidas, ya sea por segregación deficiente, almacenamiento incorrecto, recolección insegura o condiciones deficientes de almacenamiento final, las cuales pueden generar riesgos a la salud de las personas y al medio ambiente. La Figura 1 ilustra las actividades carentes de control.

\section{DisCUSIÓN}

Con respecto a la cantidad de residuos peligrosos generada, Chile no dispone de antecedentes públicos. La cifra encontrada de $0,7 \mathrm{~kg} / \mathrm{cama}$ /día, se encuentra dentro de los rangos estimados por la Organización Mundial de la Salud (OMS) para la producción de residuos peligrosos en los países de América Latina. La OMS refiere que en los centros de atención de salud en América Latina se produciría alrededor de $3 \mathrm{~kg} / \mathrm{cama} /$ día de residuos, y que sólo $10 \%$ a $25 \%$ de ellos tendrían características de peligrosidad ${ }^{4}$.

El manejo centrado en la minimización de los riesgos directos de contaminación cruzada, y daño por manipulación del personal de salud, es una primera etapa muy necesaria, pero no suficiente para asegurar que la gestión del hospital resulte saludable y amigable con el medio ambiente. $\mathrm{Si}$ bien el modelo de análisis usado en el estudio permite identificar aquellas unidades de mayor riesgo, y los procedimientos riesgosos presentes en el manejo actual de los residuos del centro hospitalario, lo que permitiría focalizar en ellos acciones de corrección para mejorar el rendimiento general del sistema, hacer solamente esto parece insuficiente. Tomando en consideración las sugerencias establecidas por la OMS para un 


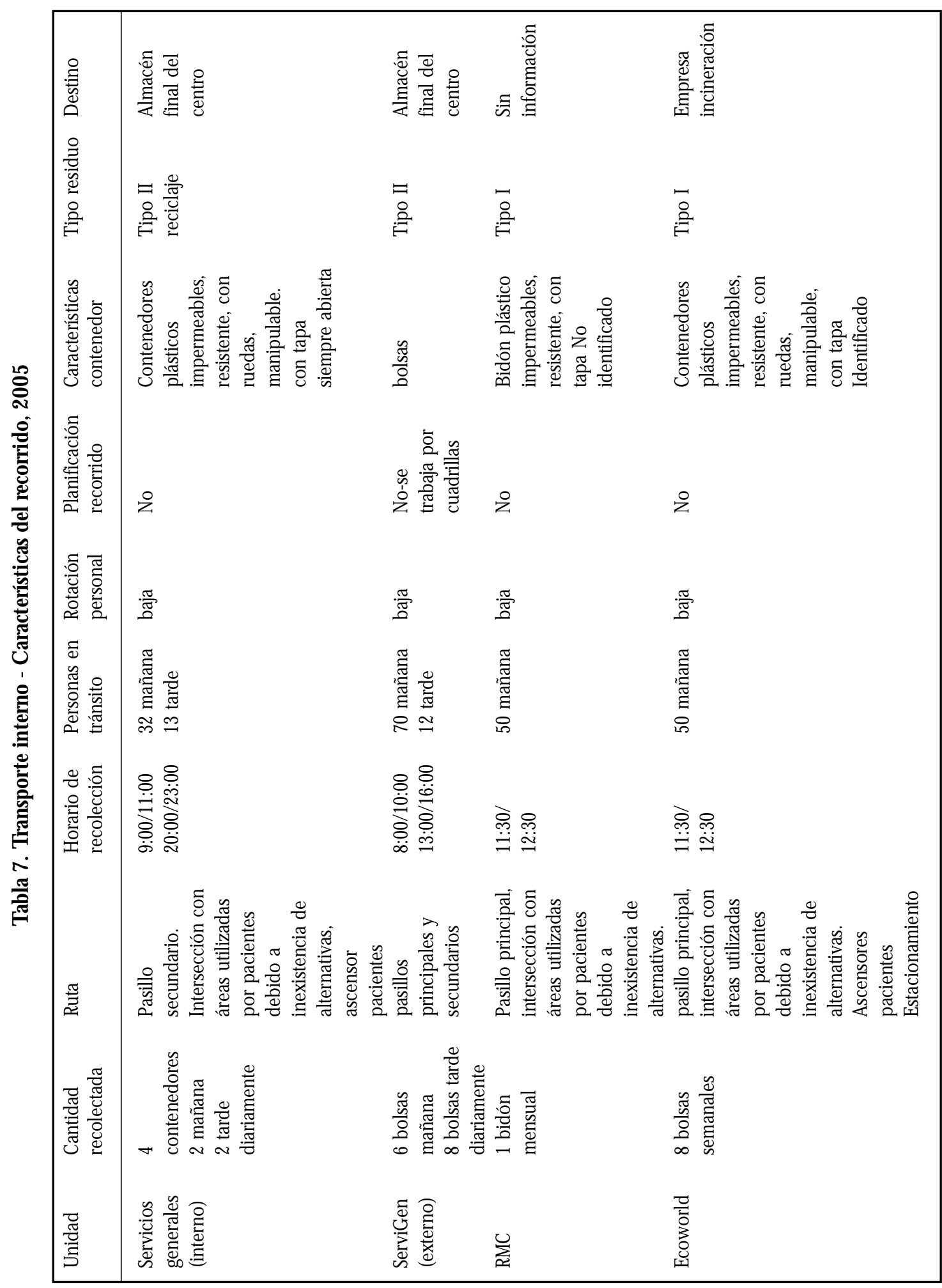


Tabla 8. Riesgo ambiental acumulado de residuos tipo I en unidades estudiadas, 2005

\begin{tabular}{|lcr|}
\hline Unidad & $\begin{array}{c}\text { Riesgo ambiental } \\
\text { acumulado por unidad }\end{array}$ & Categoría \\
\hline Anatomía patológica & 6 & Riesgo medio \\
Quimioterapia & $<$ & Riesgo bajo \\
Farmacia central & $<5$ & Riesgo bajo \\
Farmacia preparación drogas oncológicas & 7 & Riesgo alto \\
Farmacia preparación drogas & 7 & Riesgo alto \\
Farmacia unidad de dispensación & $<5$ & Riesgo bajo \\
Laboratorio & - & No dispone tipo I \\
Quimioterapia ambulatoria & $<5$ & Riesgo bajo \\
Intermedio & $<5$ & Riesgo bajo \\
Hospitalización mujeres & 6 & Riesgo medio \\
Imagenología & - & No dispone tipo I \\
Alimentación & - & No dispone tipo I \\
Braquiterapia & $<5$ & Riesgo bajo \\
Esterilización & - & No dispone tipo I \\
Policlínico & $<5$ & Riesgo bajo \\
Radioterapia & $<5$ & Riesgo bajo \\
Acupuntura & $<5$ & Riesgo bajo \\
Cirugía menor & $<5$ & Riesgo bajo \\
Endoscopia & $<5$ & Riesgo bajo \\
Cirugía & $<5$ & Riesgo bajo \\
\hline
\end{tabular}

adecuado manejo de los residuos hospitalarios, se sugiere establecer un sistema integrado de manejo de los residuos hospitalarios con énfasis en la capacitación del personal y el establecimiento de procedimientos escritos para el transporte interno de los residuos en particular de los residuos peligrosos.

En particular, si bien la costumbre de eliminar los residuos cortopunzantes en caja rígida junto con los residuos sólidos urbanos, es una acción que reduce el riesgo para los manipuladores de los residuos (personal médico y personal de aseo), al no contar el país con un sistema que asegure la adecuada disposición de residuos urbanos (vertederos ilegales y sellado diario insuficiente), en aquellas localidades en que la actividad de rastrojear basura doméstica persiste, esta modalidad de disposición puede resultar poco segura.

Finalmente, teniendo en mente las futuras exigencias que establecerá la autoridad sanitaria, explicitadas en el reglamento sobre manejo de residuos de establecimientos de atención de salud ${ }^{5}$, el Instituto debería formular e implementar un Plan de Manejo de Residuos Peligrosos e implementar las modificaciones estructurales requeridas por dicho cuerpo normativo.

El establecimiento definitivo de dicha regulación, no sólo beneficiará la gestión intema de las instalaciones de salud, asegurando un manejo seguro de los residuos, disminuyendo consecutivamente los riesgos clínicos» de contaminación directa, contaminación cruzada e infecciones hospitalarias; sino que aportará un beneficio general favoreciendo la protección ambiental de los medios potencialmente impactados (aire, suelo y aguas) por su manejo inadecuado.

\section{CONCLUSIÓN}

El Instituto evaluado genera una cantidad de residuos hospitalarios peligrosos dentro del marco de los descritos a nivel internacional. Si bien existe un sistema de gestión de ellos, con personal propio y empresas externas encargadas del retiro y disposición final, no se visualiza una gestión integral de los residuos hospitalarios. 


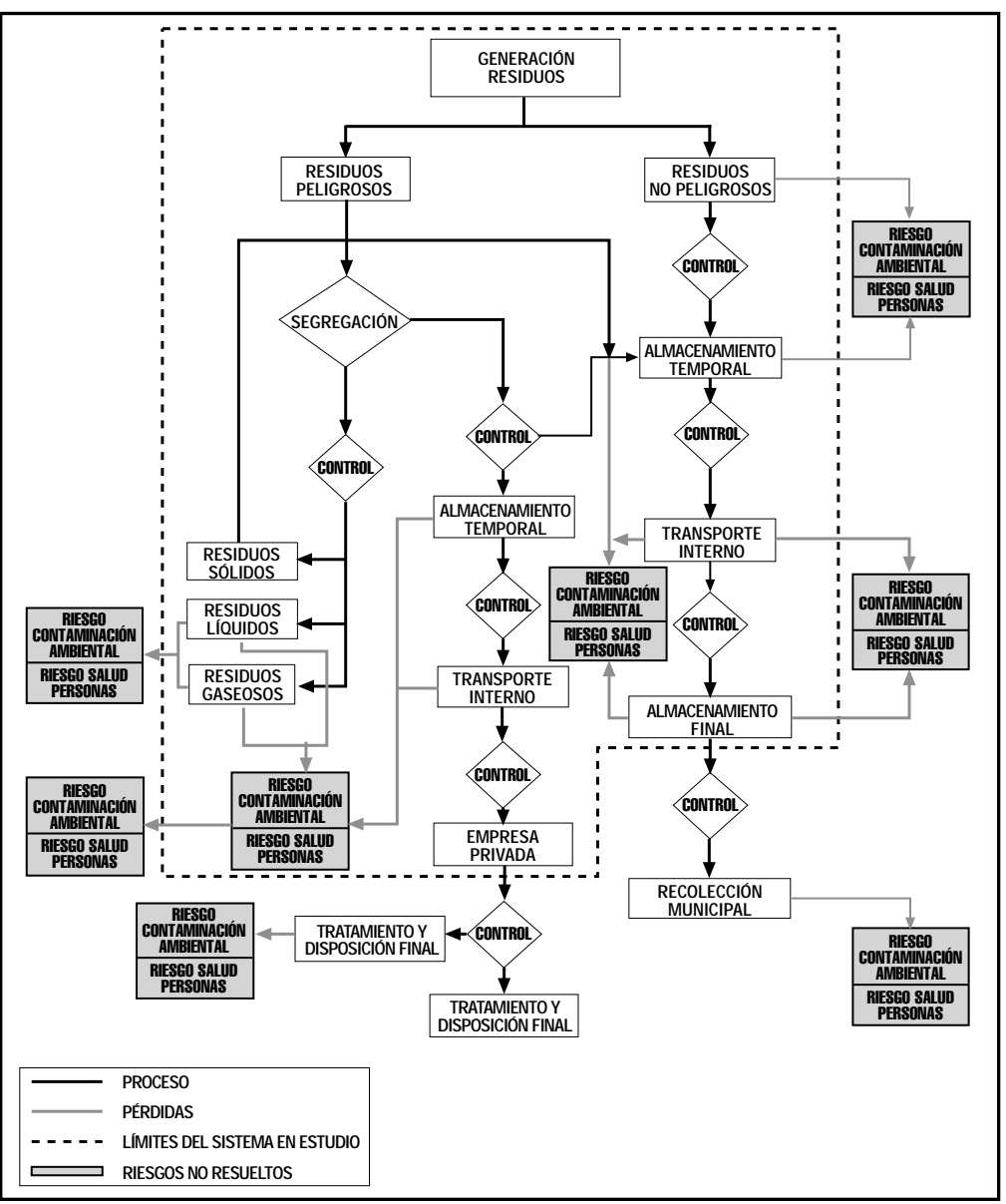

Figura 1. Modelo de manejo actual de residuos hospitalarios, 2005.

\section{REFERENCIAS}

1. Cantanhede A. La gestión y tratamiento de los residuos generados en los centros de atención de salud, CEPIS Publicaciones, Lima 1997.

2. LyNN F, Busemberg G. Citizen advisory committee and environmental policy: what we know, what's left to discover. Risk Analysis 1995; 15: 47-62.

3. HADDEN S. Public perception of hazardous waste. Risk Analysis 1991; 11: 47-57.

4. WHO Safe Management of waste from healthcare activities, Ed Pruss, Giroult \& Rushbrook, ISBN 924154525 9, 1999.
5. Ministerio DE SALUd. Aprueba reglamento sobre manejo de residuos de establecimientos de atención de salud (REAS). Documento para observaciones, página web: minsal.cl.

6. Unión EuRopea. Directiva 96/82/CE del Consejo 9 de diciembre 1996: Relativa al control y riesgos inherentes a los accidentes graves en las que intervengan sustancias peligrosas, 1997.

7. ESPAÑa, Ministerio de INDUSTRIA y ENERgía, Norma española experimental: UNE 150008 Ex: Análisis y evaluación del riesgo medioambiental. AENOR, 2002. 